\title{
Editorial for Special Issue on Intelligent Control and Computing in Advanced Robotics
}

Artificial intelligence (AI), robotics, and intelligent systems are increasingly penetrating our society. With recent advances in AI, especially in intelligent control and computing, robots are gaining the ability to learn, make decisions, and operate in ways much similar to humans. To a large extent, intelligent control can be viewed as a class of control methods which imitate human intelligence in learning, decision-making and problem solving. In the meanwhile, advances in sensors, actuators, communication and computation provide a solid foundation for the implementation of intelligent control hardware and software for a multitude of robots. In continuous endeavor to nurture robotics innovation, the synergistic integration of intelligent control hardware and software will offer ample prospects for industrial robots, mobile robots, service robots and specialized robots.

This special issue aims to discuss some of the opportunities and challenges in the design, control and applications of robots and mechatronic systems using intelligent control and computing methods and techniques. Perspectives and future directions of promoting intelligence-driven robotics research are also discussed. Undoubtedly, intelligent control and computing will play an even more important role in intelligent manufacturing, Internet of things, healthcare and intelligent services. We solicited via this special issue manuscripts in the important fields of intelligent control and computing that explore the synergy of robot-related control methods and techniques. In the end, seven papers consisting of two review papers and five research papers were accepted, which formed a special section.

The first paper, entitled "Software for small-scale robotics: a review" from Tobias Tiemerding and Sergej Fatikow, reviews current approaches for small-scale robotics (SSR). In the framework of SSR, existing software is divided into four major categories: vendor-provided software, robotic software frameworks, scientific software and in-house developed software. Each category is evaluated with respect to multiple criteria, and advantages and disadvantages are discussed.

The second paper, entitled "Current researches and future development trend of intelligent robot: a review"

\footnotetext{
Editorial

Special Issue on Intelligent Control and Computing in Advanced Robotics

Manuscript received July 13, 2018; accepted July 15, 2018

(C) Institute of Automation, Chinese Academy of Sciences and Springer-Verlag Gmbh Germany, part of Springer Nature 2018
}

from Tian-Miao Wang et al., reviews current research on key and leading technologies such as human-robot collaboration, driverless technology, emotion recognition, braincomputer interface, bionic software robot and cloud platform, and big data network. In particular, perspectives on future developments and applications of intelligent robots in China are offered in depth, shedding light on formulating future-oriented strategies and supporting the development in the era of intelligent robots.

The third paper, entitled "Rotorcraft having a 3DOF rigid manipulator: quaternion-based modeling and realtime control tolerant to multi-body couplings" from J. U. Alvarez-Muñoz et al., reports a solution to stabilization of a mini-quadcopter system with a $3 \mathrm{DoF}$ manipulator robot. A control law is presented to asymptotically stabilize the attitude and position of the system. Experimental results demonstrate the effectiveness of the proposed control law for counteracting with disturbances.

The fourth paper, entitled "A selective attention guided initiative semantic cognition algorithm for service robot" from Huan-Zhao Chen et al., presents a selective attention guided initiative semantic cognition algorithm in intelligent space. Aiming at higher adaptability and service efficiency, the authors utilize a combined method synthesizing selective attention model, recognition, knowledge storage and semantic reasoning. Furthermore, a set of experiments in real scenario verify the selective attention guided initiative semantic cognition algorithm.

The fifth paper, entitled "An energy-based nonlinear coupling control for offshore ship-mounted cranes" from $\mathrm{Yu}$-Zhe Qian et al., investigates the control problem of offshore ship-mounted cranes with intrinsic underactuated property and unmatched external disturbances. On the basis of the coupling model, a novel energy-based nonlinear controller is developed, then Lyapunov techniques as well as LaSalle's invariant theorem are coherently utilized to analyze the asymptotic stability of the closed-loop crane system. Simulations and experiments demonstrate superior control performance even under the condition of transient payload swing perturbations.

The sixth paper, entitled "Learning to transform service instructions into actions with reinforcement learning and knowledge base" from Meng-Yang Zhang et al., proposes a reinforcement learning approach aided by the knowledge base for mapping natural language instructions to executable action sequences. The authors present 
an approach to judge the result of home service operation by taking object states as evaluation factors in the context of reinforcement learning. In particular, the knowledge base is exploited as an associative mean for reinforcement learning implementation. Experimental results demonstrate great performance in terms of accuracy.

The last paper, entitled "A 3D-printed robot hand with three linkage-driven under-actuated fingers" from Xia-Li Li et al., describe a robot palm and three-finger robot hand system. Specifically, the authors improve an under-actuated finger mechanism to simulate the grasp operation of a finger under different situations. They utilize $3 \mathrm{D}$ printing to fabricate the robot hand to reduce the cost of the production process and enhance the design flexibility. Grasping experiments on the actual robotic system show that the robot is able to grasp objects with different sizes.

Min Tan

Institute of Automation, Chinese Academy of Sciences, China

Yu Sun

University of Toronto, Canada

Jun-Zhi Yu

Institute of Automation, Chinese Academy of Sciences, China

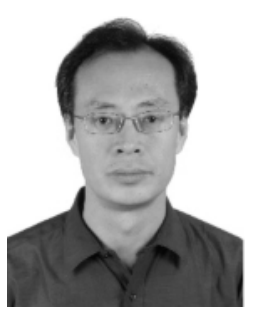

Min Tan is a professor in the State Key Laboratory of Management and Control for Complex Systems, Institute of Automation, Chinese Academy of Sciences, China.

His research interests include advanced robot control, biomimetic robot, and multirobot system.

E-mail: min.tan@ia.ac.cn

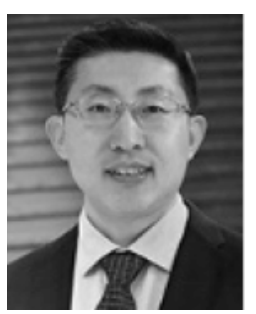

Yu Sun is a professor in the Department of Mechanical and Industrial Engineering, with joint appointments in the Institute of Biomaterials and Biomedical Engineering and the Department of Electrical and Computer Engineering at the University of Toronto, Canada. He was elected Fellow of IEEE, ASME, AAAS, and CAE for his work on micro-nano robotic systems.

His research interests include biomedical engineering, robotics, mechatronics and instrumentation.

E-mail: sun@mie.utoronto.ca

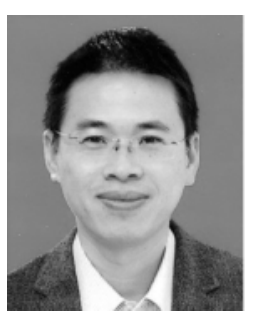

Jun-Zhi $\mathbf{Y u}$ is a professor with the State Key Laboratory of Management and Control for Complex Systems, Chinese Academy of Sciences, China. His research interests include biomimetic robots, intelligent control, and intelligent mechatronic systems.

$\mathrm{He}$ is an associate editor of the IEEF Transactions on Robotics and the IEEE/ASME Transactions on Mechatronics.

E-mail: junzhi.yu@ia.ac.cn (Corresponding author) ORCID iD: 0000-0002-6347-572X 